\title{
In Focus
Spotlight on the October 8 Issue
}

\author{
Robert A. Gross, MD, PhD, FAAN \\ Editor-in-Chief, Neurology ${ }^{\circledR}$
}

\section{Randomized trial of cognitive speed of processing training in Parkinson disease $\underline{\underline{\underline{A}}}$}

Eighty-seven adults with a diagnosis of idiopathic Parkinson disease and on a stable medication regimen participated in either 20 hours of self-administered speed of processing training or a no-contact control condition. Speed of processing training improved Useful Field of View Test performance among persons in the mild to moderate stages of Parkinson disease.

See p. 1284; Editorial, p. 1278

Vitamin B supplementation, homocysteine levels, and the risk of cerebrovascular disease: A meta-analysis

The authors examined the association between $B$ vitamin supplementation and endpoint events using a fixed-effects model on 54,913 participants. B vitamin supplementation for homocysteine reduction reduced stroke events, especially in subjects with certain characteristics who received appropriate intervention measures.

See p. 1298

Migraine and white matter hyperintensities: The ARIC MRI study White matter hyperintensities (WMH) are often attributed to migraine in younger patients and to vascular risk factors in older patients. In this study, migraine was associated with baseline $\mathrm{WMH}$ volume but not with greater progression in older age. Migraine is more likely to affect white matter earlier in life.

See p. 1308

Episodic migraine and obesity and the influence of age, race, and sex The odds ratio of episodic migraine was increased by $81 \%$ in those with obesity. Additionally, age-stratified results showed that the risk of migraine was stronger in those younger than 50 than in those 50 or older. Clinicians should educate their patients that obesity increases the risk of episodic migraine and should choose medications with consideration for impact on patients' weight, as well as advise healthy diet and exercise lifestyles.

See p. 1314
Anterior brain glucose hypometabolism predates dementia in progranulin mutation carriers

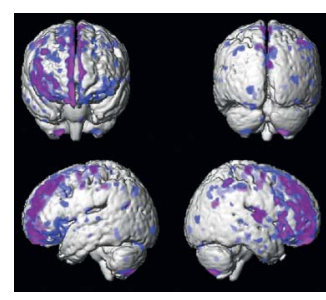

Nine mutation carriers and 11 noncarriers from 5 families with frontotemporal dementia (FTD) underwent brain scanning and clinical evaluation. Carriers of progranulin mutations showed an abnormally low anterior-to-posterior uptake ratio, which was evident before clinical onset. FDG-PET may help diagnose and monitor some forms of familial FTD. See p. 1322

From editorialists Rabinovici \& Boeve: "A better understanding of the clinical and imaging features that truly define 'prodromal' FTD is critical for early diagnosis and for future clinical trial design. New diagnostic criteria that include a possible FTD category may be an important starting point."

See p. 1282

Progression to dementia in memory clinic patients without dementia: A latent profile analysis

In 635 patients without dementia, latent profile analysis identified cognitive subtypes based on immediate and delayed memory recall, delayed recognition, information-processing speed, attention, verbal fluency, and executive functions. Even mild memory impairment might flag individuals at high risk for dementia if nonmemory deficits are present.

See p. 1342

Analysis of start-up, retention, and adherence in ALS clinical trials Analysis of start-up times, retention, and protocol adherence was performed on 5 clinical studies and 50 ALS clinical trials. Delays in institutional review board review contributed the most to prolonged trial start-up times, and these timelines were faster in sites with more experienced staff. Strategies to improve protocol adherence and participants' retention should include enrollment at earlier disease stages. See p. 1350 


\section{Neurology}

Spotlight on the October 8 Issue

Robert A. Gross

Neurology 2013;81;1277

DOI 10.1212/WNL.0b013e3182a988d3

\section{This information is current as of October 7, 2013}

\section{Updated Information \&} Services

Permissions \& Licensing

Reprints including high resolution figures, can be found at: http://n.neurology.org/content/81/15/1277.full

Information about reproducing this article in parts (figures,tables) or in its entirety can be found online at:

http://www.neurology.org/about/about_the_journal\#permissions

Information about ordering reprints can be found online:

http://n.neurology.org/subscribers/advertise

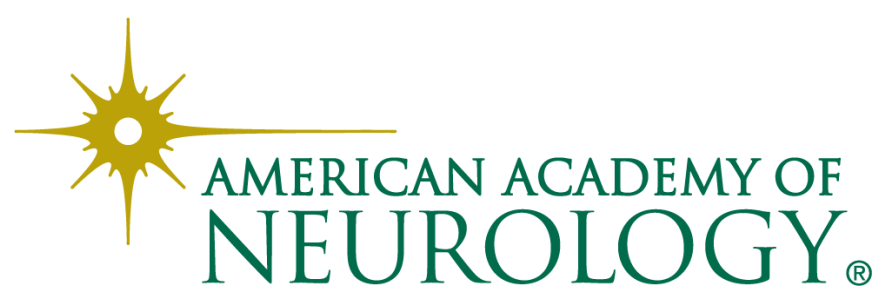

